\title{
Pinning and gyration dynamics of magnetic vortices revealed by correlative Lorentz and bright-field imaging
}

\author{
Marcel Möller $\odot,{ }^{*}$ John H. Gaida $\odot$, and Claus Ropers $\odot^{\dagger}$ \\ Max Planck Institute for Multidisciplinary Sciences, 37077 Göttingen, Germany \\ and 4th Physical Institute - Solids and Nanostructures, \\ University of Göttingen, 37077 Göttingen, Germany
}

(Received 22 July 2021; accepted 2 December 2021; published 11 January 2022)

\begin{abstract}
Topological magnetic textures are of great interest in various scientific and technological fields. To allow for precise control of nanoscale magnetism, it is of great importance to understand the role of intrinsic defects in the host material. Here we use conventional and time-resolved Lorentz microscopy to study the effect of grain size in polycrystalline permalloy films on the pinning and gyration orbits of vortex cores inside magnetic nanoislands. To assess static pinning, we use in-plane magnetic fields to shift the core across the island while recording its position. This enables us to produce highly accurate two-dimensional maps of pinning sites. Based on this technique, we can generate a quantitative map of the pinning potential for the core, which we identify as being governed by grain boundaries. Furthermore, we investigate the effects of pinning on the dynamic behavior of the vortex core using stroboscopic Lorentz microscopy, harnessing a new photoemission source that accelerates image acquisition by about two orders of magnitude. We find characteristic changes to the vortex gyration in the form of increased dissipation and enhanced bistability in samples with larger grains.
\end{abstract}

DOI: 10.1103/PhysRevResearch.4.013027

\section{INTRODUCTION}

Microscopic magnetic objects such as vortices [1-3] and skyrmions [4-6] have attracted a sustained interest in the past decade. Due to their stability and unique topological properties, these textures have sparked ideas for a vast number of technological applications such as (racetrack) memories [7-10], logical-gates [11,12], and neuromorphic computing [13]. While various control schemes for the manipulation of these textures by means of external magnetic fields [14,15], electrical currents [16-18], or optical pulses [3,18,19] are widely established, studies probing their interaction with defects are still highly sought after.

Previous investigations addressed the influence of artificial [20-23] and intrinsic [24-30] defects on the static pinning $[20,21,23,24]$ and the dynamics of magnetic vortices $[22,25-$ 28,31]. A majority of these studies utilized magneto-optical microscopy [25-29], which helped, for example, to link pinning to surface roughness in soft magnetic samples [27]. Higher real-space resolution is offered by spin-polarized scanning tunneling microscopy in scenarios with atomic-scale defects on flat surfaces $[23,32]$. This method revealed a Sombrero-shaped pinning potential between vortex cores and surface adsorbates [23]. Excellent spatial resolution for poly-

*marcel.moeller@uni-goettingen.de

†claus.ropers@mpinat.mpg.de

Published by the American Physical Society under the terms of the Creative Commons Attribution 4.0 International license. Further distribution of this work must maintain attribution to the author(s) and the published article's title, journal citation, and DOI. Open access publication funded by the Max Planck Society. crystalline samples with higher surface roughness is possible using electron microscopy techniques [33] and was used to characterize core pinning via one-dimensional differentialphase-contrast line scans [21]. Moreover, recent advances in time-resolved Lorentz microscopy enable imaging magnetic dynamics at simultaneous high spatial and temporal resolution [34-39]. To date, however, the full capabilities of electron microscopy in both magnetic and structural imaging have yet to be leveraged in correlated studies.

In this work we investigate the influence of the microcrystalline structure of permalloy thin films on the pinning of vortex cores by correlating the grain structure in bright-field images to the magnetic configuration in Lorentz micrographs. In order to obtain maps of pinning sites, we developed TRaPS (TEM Rastering of Pinning Sites). This procedure locates defects by laterally shifting the vortex core across a nanostructure and imaging its position with high resolution. This allows us to directly calculate a quantitative two-dimensional representation of the pinning potential. Moreover, we use time-resolved Lorentz microscopy with an improved photoemission source to assess the effect of grain size on the core gyration. We find that annealing leads to a more corrugated pinning potential and larger average distances between pinning sites. Our findings suggest preferential pinning at grain boundaries and vortex orbits that avoid particularly large grains, demonstrating the combined strengths of correlated and in situ magnetic and structural characterization.

\section{METHODS}

\section{A. Sample system}

The sample system we investigate is a magnetic vortex confined in a square permalloy $\left(\mathrm{Ni}_{80} \mathrm{Fe}_{20}\right)$ nanoisland [1,2]. A 


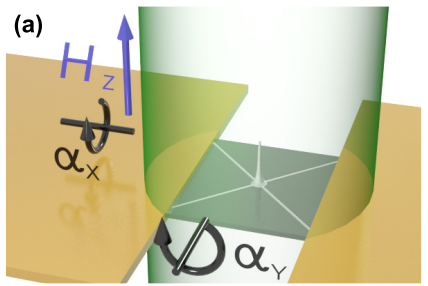

(c)
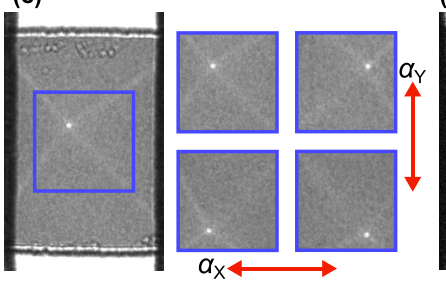

(d)
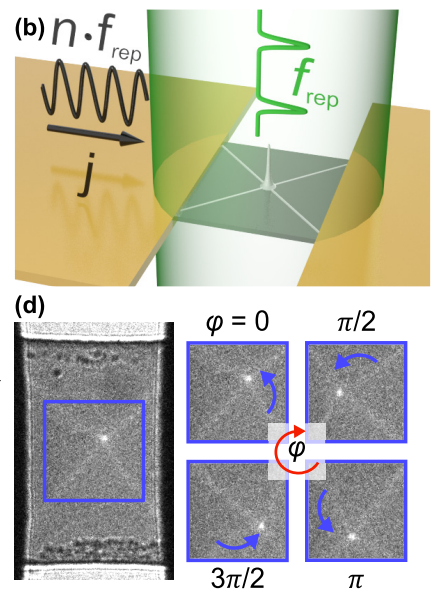

FIG. 1. (a) Sketch of the TRaPS measurement: The magnetic nanoisland (dark gray) is illuminated with a continuous collimated electron beam (green) and subjected to an external magnetic field $H_{z}$. Tilting the sample along the two tilt axes $\alpha_{x}$ and $\alpha_{y}$ enables us to move the vortex core within the nanostructure. (b) Sketch of the time-resolved experimental setup: Here the nanoisland is imaged using a pulsed electron beam at a repetition rate of $f_{\text {rep }}$ and excited with a synchronized alternating current at an integer multiple of the frequency of $f_{\text {ex }}=n f_{\text {rep }}$. (c) Four micrographs illustrating the displacement of the core during a TRaPS measurement. A tilt along $\alpha_{x}$ or $\alpha_{y}$ has the same effect as applying an in-plane field $H_{y}$ or $H_{x}$, respectively. (d) Example of four time-resolved Lorentz micrographs acquired at different phases $\varphi$ of the exciting current relative to the pulsed electron beam. The bright lines indicate the position of the domain walls within the nanostructure, with their intersection marking the location of the vortex core. Between frames, the core is visibly displaced and moving on a counterclockwise trajectory.

magnetic vortex is a flux-closure type domain configuration which is predominantly oriented in-plane and curls either counterclockwise $(c=+1)$ or clockwise $(c=-1)$ around its central core. At the core region, the magnetization rotates out-of-plane and, either points up or down, said to have a polarization of $p=+1$ or $p=-1$, respectively [2].

A schematic representation of the sample is depicted in Figs. 1(a) and 1(b) (light and scanning-electron images of the sample are in the Supplemental Material Fig. 5 [40]). The permalloy square has a thickness of $30 \mathrm{~nm}$ and an edge length of $2 \mu \mathrm{m}$. To electronically excite the sample, we overlap $100 \mathrm{~nm}$ thick gold contacts on two opposing sides of the square, which extend to wire-bonding pads. The nanoisland is positioned at the center of a $15 \mu \mathrm{m} \times 15 \mu \mathrm{m}$ large amorphous silicon nitride window. At a thickness of $30 \mathrm{~nm}$ the window is near electron transparent and is supported by a $200 \mu \mathrm{m}$ thick silicon frame.

The sample fabrication processes involve electron-beam lithography using a positive-tone electron resist as well as electron-beam and thermal evaporation for the deposition of permalloy and gold, respectively. We take special care to remove any resist prior to metal deposition by subjecting the developed sample to a short oxygen plasma.

The contacts on either side of the microstructure allow us to excite the vortex with in-plane RF currents, forcing its core on an elliptical trajectory [41-43]. This gyrotropic motion

is a consequence of a combination of spin-transfer torques and current-induced Oersted fields [42,44]. Generally these systems allow for a resonance frequency between $10 \mathrm{MHz}$ and $10 \mathrm{GHz}$, depending on the material and nanostructure size. For the parameters of the current sample, we expect resonance frequencies around $100 \mathrm{MHz}$ [37].

To identify the effects of the nanocrystalline structure on the pinning of vortex core, we investigate one annealed sample $A$ as well as two nonannealed samples $\mathbf{N 1}$ and $\mathbf{N 2}$. We prepared all three samples under identical conditions on the same silicon frame. Annealing is carried out by heating a single nanostructure using a low-frequency alternating current $\left(5 \mathrm{kHz}, 450 \mathrm{mV}_{\mathrm{rms}}, 4.9 \mathrm{~mA}_{\mathrm{rms}}, 30 \mathrm{~min}\right)$ and monitoring the progress via a resistance measurement (for further details, see [40]).

\section{B. TRaPS}

We introduce TRaPS as a method to identify and locate static magnetic pinning sites using Fresnel-mode Lorentz microscopy. This imaging method is sensitive to a curl of the in-plane components of the magnetization and offers a point resolution on the order of some tens of nanometers, depending on the imaging conditions such as defocus and source coherence $[45,46]$. In order to minimize the magnetic field at the sample, for Lorentz imaging, the main objective lens is usually turned off, and a minilens farther from the sample is used to produce the image. For the vortex samples studied here, Fresnel imaging visually highlights the position of the four domain walls as bright lines [cf. Fig. 1(c)]. At their intersection, a peak is formed, marking the position of the vortex core. Although this peak is not a direct consequence of the out-of-plane magnetization of the core, both properties are geometrically linked. As we note below, we can localize the core with a precision far below the point resolution of the imaging technique [37]. To perform a TRaPS measurement, we shift the core across the film in a rasterized fashion using in-plane magnetic fields and record Lorentz micrographs at each raster step. A repeated occurrence of the core at the same position can then indicate the location of a pinning site.

We generate the in-plane magnetic field components $H_{x}$, $H_{y}$ along the $x$ and $y$ directions of the sample by applying an out-of-plane field $H_{z}$ and tilting the sample along the two tilt axes $\alpha_{x}, \alpha_{y}$, as indicated in Figs. 1(a) and 1(c). For sufficiently small angles, the resulting field components are $\left(H_{x}, H_{y}\right) \approx H_{z}\left(\alpha_{y}, \alpha_{x}\right)$. We create the out-of-plane magnetic field $H_{z}$ by weakly exciting the main objective lens of the microscope. This introduces a small additional defocus to the imaging system, which can easily be compensated for with the objective minilens.

\section{Time-resolved Lorentz microscopy}

Time-resolved Lorentz microscopy is carried out at the Göttingen ultrafast transmission electron microscope (UTEM), a modified JEOL $2100 \mathrm{~F}$ transmission electron microscope (TEM) featuring a high-brightness photoemission electron source $[37,47]$. For the present study we equipped the UTEM with a radio-frequency $(\mathrm{RF})$ generator that is electronically synchronized to the photoemission laser, using a 
methodology introduced in Ref. [37]. One output of the RF generator (Keysight 81160A) feeds into a custom TEM holder that enables TEM imaging under in situ current excitation with frequencies up to the $\mathrm{GHz}$ regime. A second output triggers the photoemission laser, a gain-switched diode laser (custom Onefive Katana 05-HP) operating at a wavelength of $532 \mathrm{~nm}$. With its continuously variable repetition rate of $f_{\text {rep }}=20-80 \mathrm{MHz}$ and a pulse duration of $35 \mathrm{ps}$, this laser enables us to increase the electron-pulse duty cycle by more than two orders of magnitude compared to previous studies [37]. Thus, we can reduce the image acquisition time of a single stroboscopic micrograph from several minutes to a few seconds and compile much larger data sets that allow deeper insights into the vortex gyration.

We compile time-resolved movies of the vortex dynamics by exciting it at frequencies that are integer multiples of the laser repetition rate $f_{\text {ex }}=n f_{\text {rep }}$ and by incrementally changing the excitation phase between frames [see Figs. 1(b) and 1(d)].

\section{Bright-field imaging and vortex core localization}

To assess the nanocrystalline structure of our samples, we recorded bright-field images in low-magnification mode [Figs. 2(a) and 2(b)] by filtering out scattered parts of the beam using an aperture. In bright-field imaging, contrast variations in a film arise from spatially varying diffraction conditions of differently oriented grains. This allows us to compare the vortex core positions we find in time-resolved and TRaPS measurements to the grain structure of the films. Therefore, we track the core in the Lorentz micrographs and map the results on top of the bright-field images. The tracking process involves calculating the center-of-mass of the pixels corresponding to the bright peak at the core position, which we identify as the largest pixel cluster above an intensity threshold. This method enables a core localization with fewnanometer precision, well below the typical point resolution of Lorentz microscopy (usually tens of nanometers) [37]. To map the core positions onto the bright-field images, we use a geometric transformation [48], which is derived from the location of easily identifiable image features in both Lorentz and bright-field images. Using this approach, we can translate position information between both reference frames.

\section{RESULTS}

\section{A. TRaPS measurements}

TRaPS measurements are performed on the nonannealed sample N1 and on sample A. In their bright-field images [Figs. 2(a) and 2(b)], the bright and dark regions surrounding the gray permalloy nanostructure are bare silicon-nitride and the opaque gold contacts, respectively. A thin residue of permalloy, stemming from a partially coated undercut of the electron beam resist, is faintly visible at the top and bottom of the squares and can also be seen in the Lorentz images [e.g., Fig. 1(d)]. The bright-field images in Figs. 2(c) and 2(d) reveal a significant increase in grain size in the annealed sample $\mathbf{A}$ over the nonannealed sample N1. This change in grain size is less significant in the vicinity of the gold contacts as these locally increase the thermal coupling, resulting in
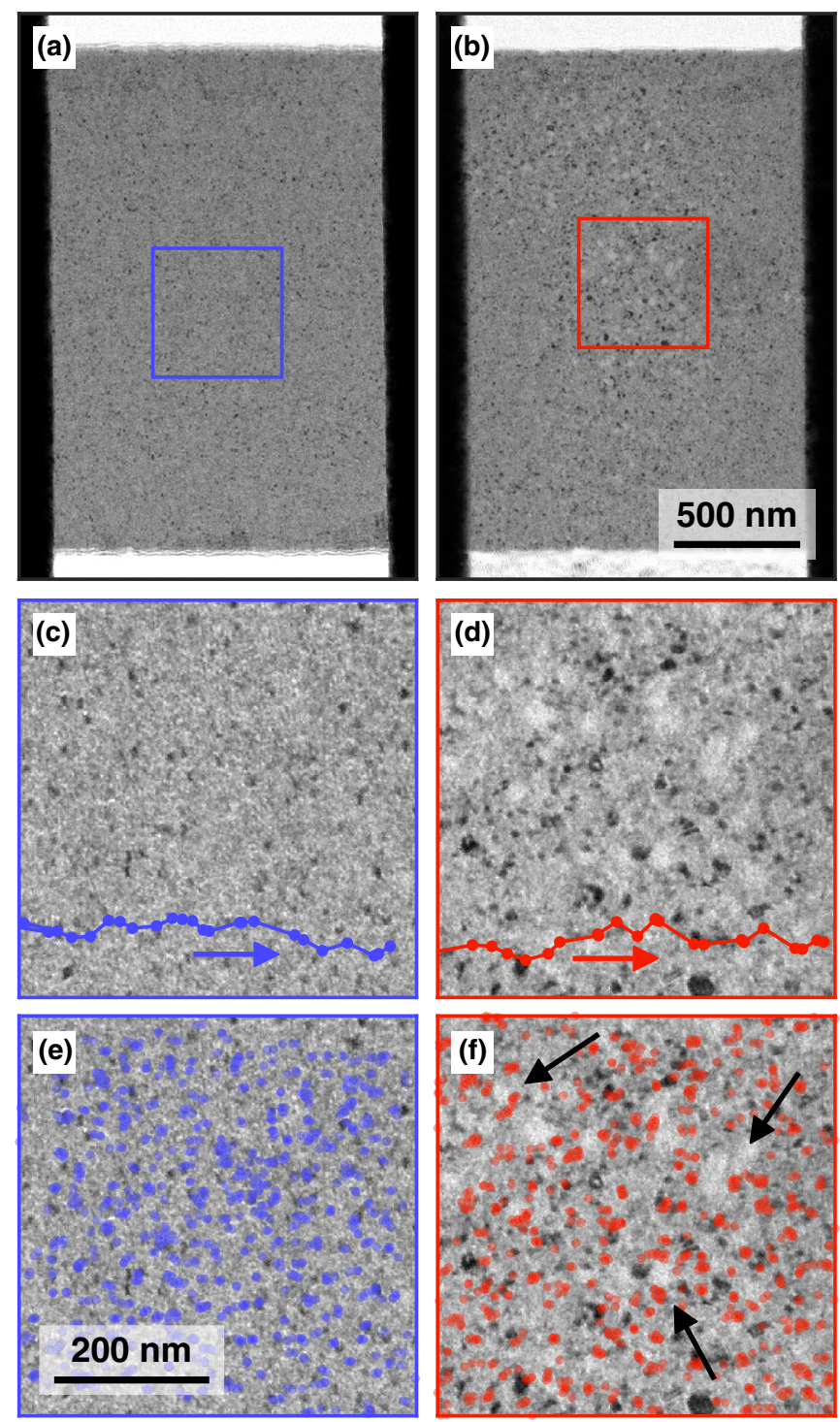

FIG. 2. Bright-field images of the nonannealed sample N1 (a) and the annealed sample $\mathbf{A}$ (b). (c) and (d) Close-ups of the regions marked in (a) and (b). The annealed sample shows a drastically increased grain size in the depicted area. The lines indicate the path of the vortex core during an exemplary tilt of primary tilt axis $\alpha_{x}$, while $\alpha_{y}$ is kept constant. (e) and (f) Tracked vortex core positions overlaid on top of the bright-field images in (c) and (d). A single tracked position is plotted with a partial transparency, such that if the core is encountered at the same location repeatedly, the superimposed dots appear more saturated and opaque. An alternative depiction of the data in (e) and (f) is given in the Supplemental Material Fig. 6 indicating the angle of the slow tilt axis $\alpha_{y}$ [40].

an inhomogeneous temperature profile during the annealing process.

For the TRaPS measurements we apply an external field of $H_{z}=35.8 \mathrm{kAm}^{-1}$ and vary both tilt axes in a range from $-2.2^{\circ}$ to $2.2^{\circ}$ in increments of $0.2^{\circ}$. This corresponds to a change in-plane field of about $620 \mathrm{~A} \mathrm{~m}^{-1}$ per $1^{\circ}$ of tilt. For these small tilt angles, we do not expect a significant contribution from the out-of-plane magnetization of the vortex core to affect its localization. For comparison, in Ref. [49], a tilt from 
$+30^{\circ}$ to $-30^{\circ}$ shifted the peak of the Lorentz contrast at the core position by about $10 \mathrm{~nm}$. Along the primary tilt direction $\alpha_{x}$ the samples are tilted back and forth once for every tilt position of the secondary axis $\alpha_{y}$. Additionally, each sweep of $\alpha_{x}$ includes an approach step at either $\alpha_{x}=3.0^{\circ}$ or $-3.0^{\circ}$. Supplementary Movies 1 and 2 show, apart from the approach steps, the complete TRaPS measurements of either sample, with some example micrographs of sample N1 presented in Fig. 1(c) [40].

As an example we marked the path of the core during one sweep of $\alpha_{x}$ in Figs. 2(c) and 2(d), where we see that the core does not move in straight lines but rather zigzags and occasionally gets trapped. All tracked positions are marked with dots in Figs. 2(e) and 2(f) where they are plotted on top of the bright-field image of the corresponding region. An alternative depiction of the data is provided in the Supplemental Material Fig. 6, with the dots color coded as a function of tilt angle $\alpha_{y}$ [40]. Due to the small size of the grains in sample $\mathbf{N 1}$, there is no apparent visual correspondence between the nanocrystalline structure and the core positions [Fig. 2(e)]. However, we cannot exclude possible correlations on length scales smaller than those resolved in the bright-field images.

As for the annealed sample $\mathbf{A}$, we find numerous pinning sites located directly at boundaries of larger grains [see arrows Fig. 2(f)]. Furthermore, it stands out that the core never resides within one of the large grains. An in-depth analysis of the image intensities at the core positions is given in the Supplemental Material Note 3 and confirms these findings [40]. For sample $\mathbf{A}$ the analysis shows that the core rather resides at intermediate image intensities found outside of dark or bright grains. Together, these observations clearly demonstrate that grain boundaries can pin vortex cores in polycrystalline films. While this behavior was suspected before [29], it has, to our knowledge, never been directly observed. Interestingly, however, we cannot find direct evidence for a movement of the vortex core along large domain boundaries. This indicates that grain boundaries rather serve as a necessary than a sufficient condition for pinning, suggesting additional contributions to the final position of the pinning site.

To assess the accuracy of our measurement technique, we repeat the tracking and the transform calculation for a second set of experimental conditions on sample $\mathbf{A}$. The results presented in Fig. 2(d) are acquired with a clockwise curl $(c=-1)$, necessitating underfocused imaging conditions to achieve a bright spot at the core position [50]. For the second measurement we simultaneously alter the domain state to a counterclockwise curl $(c=+1)$, and employ overfocused imaging conditions. This serves two purposes: First, the magnetic image contrast will again appear bright, which is necessary for the core tracking algorithm to work properly [50]. Second, the electrostatic contributions in the Lorentz image will significantly change. Therefore, the process of correlating the Lorentz to the bright-field micrographs has to be repeated completely independently from the first data set, whose accuracy can thus be verified. The results for the second set of tracking data are shown in Fig. 3(a), and are in excellent agreement with the previous results. This is particularly evident when we plot both data sets together in the same reference frame as in Fig. 3(b), where the only discernible difference is a minor lateral displacement of about $6 \mathrm{~nm}$.
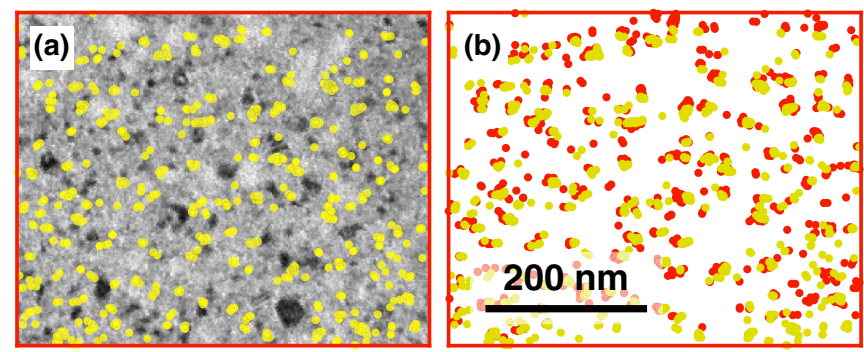

FIG. 3. Comparison between TRaPS measurement on sample A with clockwise curl $c=-1$ and underfocus imaging conditions (red dots) and counterclockwise curl $c=+1$ and overfocus imaging conditions (yellow dots). (a) Tracked vortex core position overlaid onto bright-field image of sample A. (b) Depiction of the tracked vortex position of both measurements in the reference frame of the bright-field image in (a). Both data sets are in good agreement with each other, with only a minor offset between the two. The difference in the overall center of both TRaPS measurements can be explained with a residual magnetic in-plane field at the sample position (see text).

In addition to confirming that TRaPS allows for accurate localization of pinning sites, this comparison also demonstrates that the underlying process is independent of the vortex curl.

To further analyze our data, we calculate the distance $d$ traveled by the vortex between two consecutive steps of a TRaPS scan. Figure 4(a) shows histograms of the jump distances for both samples (the initialization steps at $\alpha_{x}= \pm 3^{\circ}$ are not included in these statistics). The most prominent feature in both distributions is a strong peak at small distances $d<8 \mathrm{~nm}$. For these distances we can assume that the vortex has remained at the same pinning site, and as we would expect, this happens more frequently for sample A. In contrast, we identify substantial differences between both samples for
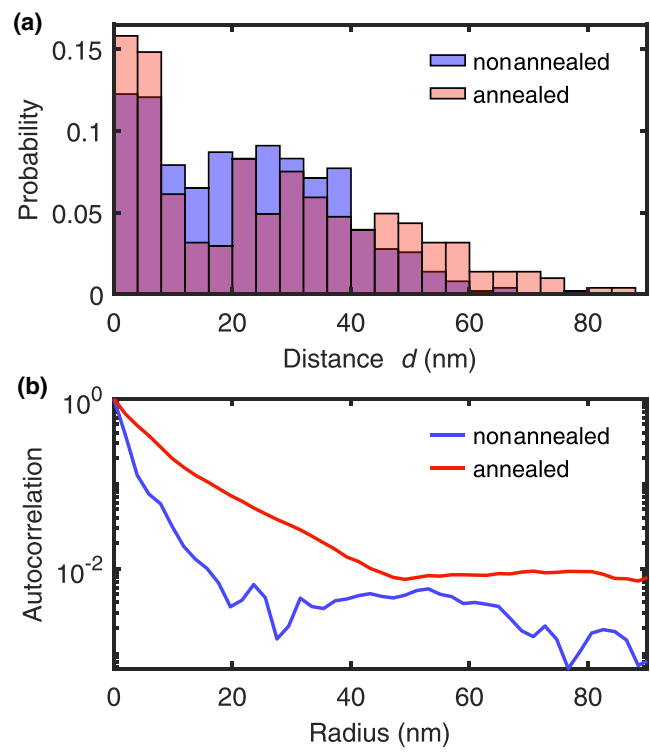

FIG. 4. (a) Probability that the vortex core moved by distance $d$ between two TRaPS steps. (b) Radial autocorrelation of the brightfield images displayed in Figs. 2(c) and 2(d). 
distances $d>8 \mathrm{~nm}$ : First, the vortex in the annealed sample rarely moves by distances in the range of 8 to $20 \mathrm{~nm}$, and second, it more frequently jumps over longer distances beyond $40 \mathrm{~nm}$, which evidently is a result of larger grain sizes.

For comparison, we also compute the average grain size in both samples using the full-width half-maximum of the radial autocorrelation function [51-53]. From the autocorrelation functions in Fig. 4(b) we estimate grain sizes of 4 and $10 \mathrm{~nm}$ for samples $\mathbf{N 1}$ and $\mathbf{A}$, respectively. This demonstrates that the typical jump distances span up to several grain diameters, suggesting that not every grain boundary causes effective pinning.

\section{B. Quantifying the pinning potential}

In the following we will detail how, based on our TRaPS data, we can quantitatively assess the pinning potential in our samples. To this end we choose a simplistic approach to obtain a two-dimensional representation of the potential as a whole (instead of treating each pinning site individually). As illustrated in Fig. 5(a), we assume that each core position $\left(X_{i, \text { expt }}, Y_{i, \text { expt }}\right)$ (gray) measured at every individual step of the TRaPS measurement $i$ contributes to the potential with an identical Gaussian dip (depth: $E_{\text {pin }}$, width: $\sigma_{\text {pin }}$ ) $[23,24,28,29]$. The overall pinning potential is then given by the sum of all Gaussians, ensuring a deeper and/or broader potential in regions where the core is encountered more frequently, and reads

$$
E_{\text {pin,all }}=-E_{\text {pin }} \sum_{i} \exp \left(-\frac{r_{i}^{2}}{2 \sigma_{\text {pin }}^{2}}\right) \text {, }
$$

where $r_{i}^{2}=\left(X-X_{i, \text { expt }}\right)^{2}+\left(Y-Y_{i, \text { expt }}\right)^{2}$ is the squared distance to the core position measured at tilt step $i$.

Under these assumptions, the pinning potential results directly from the TRaPS measurement and the two values $E_{\text {pin }}$ and $\sigma_{\text {pin }}$, which are to be determined in the following. To compare different choices of $E_{\text {pin }}$ and $\sigma_{\text {pin }}$, we perform multiple TRaPS simulations based on $E_{\text {pin,all }}$ and choose the value tuple which best reproduces the experimental data.

During a single TRaPS simulation, we replicate how the core is trapped and moves between pinning sites by scanning a quadratic potential across $E_{\text {pin,all }}$. We derive the quadratic potential from the rigid vortex model [54], for which the energy of the vortex domain configuration is expressed in terms of the core position $(X, Y)$ and the external magnetic field

$$
E_{\text {quad }}=\frac{1}{2} k\left(X^{2}+Y^{2}\right)+c k \chi\left(-H_{x} Y+H_{y} X\right) .
$$

Here $k$ is the stiffness factor of the quadratic potential, and $\chi$ is the displacement susceptibility. For our sample geometry we calculate a stiffness factor of $k=1.63 \times 10^{-3} \mathrm{eVnm}^{-2}$ using micromagnetic simulations [55]. This simulation is performed with a $512 \times 512 \times 4$ cell geometry, an exchange coupling of $A_{\mathrm{ex}}=1.11 \times 10^{-11} \mathrm{~J} \mathrm{~m}^{-1}$ [56], and a saturation magnetization of $M_{\mathrm{S}}=440 \mathrm{kA} \mathrm{m}^{-1}$ [57].

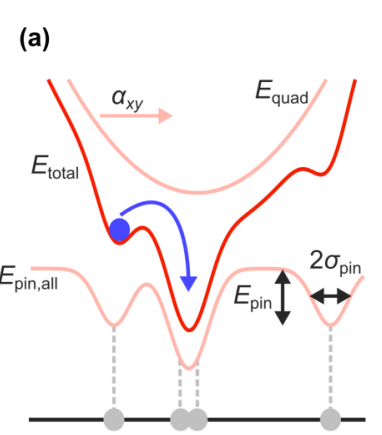

(b)
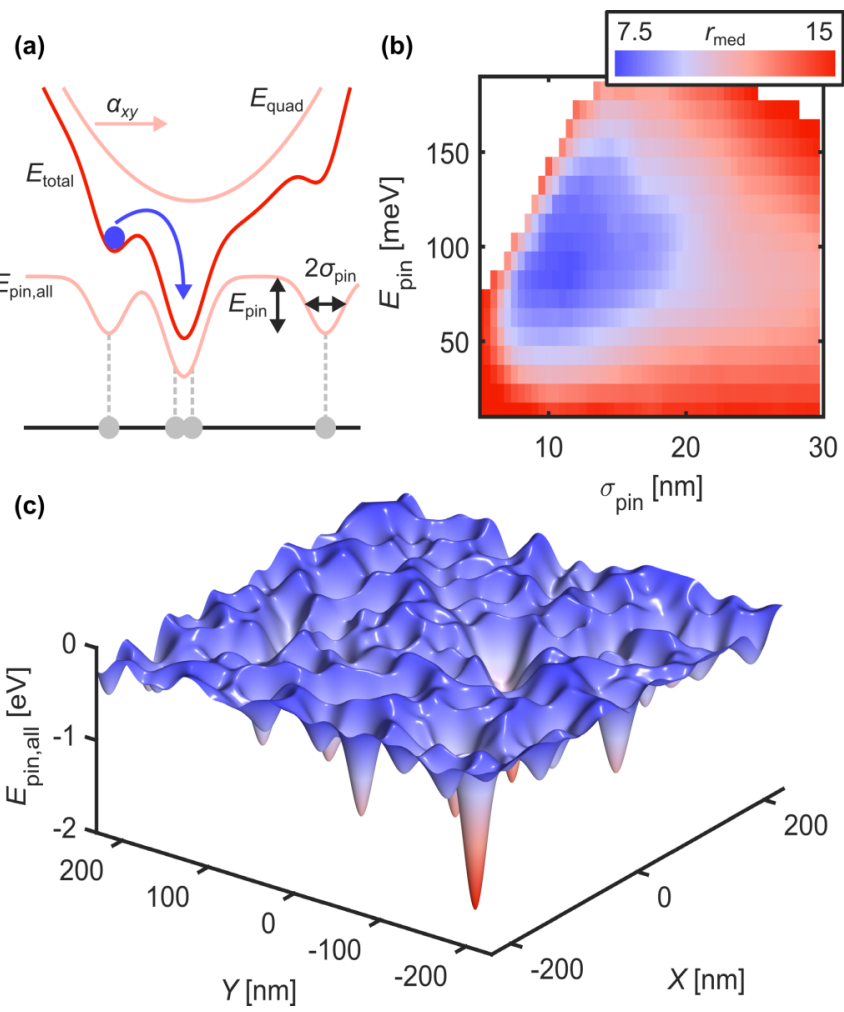

FIG. 5. Reconstruction of the pinning potential from TRaPS data of sample N1. (a) Illustration of model potential $E_{\text {total }}$ used for the simulated TRaPS data. $E_{\text {quad }}$ is a quadratic plus a field-dependent linear potential (here as a function of the tilt angle $\alpha_{x, y}$ ) that defines the equilibrium position of the vortex core in the absence of any pinning. $E_{\text {pin,all }}$ is composed of a Gaussian potential for each experimentally measured pinning position (gray dots), all having the same depth $E_{\text {pin }}$ and width $\sigma_{\text {pin }}$. By increasing/decreasing the tilt angle, the vortex core is dragged along and probes the pinning potential. (b) Median radial deviation $r_{\text {med }}$ between the experimental and simulated TRaPS data for various combinations of $E_{\text {pin }}$ and width $\sigma_{\text {pin }}$. The best agreement is obtained at $r_{\text {med }}=7.6 \mathrm{~nm}$ with $E_{\text {pin }}=80 \mathrm{meV}$ and $\sigma_{\text {pin }}=11 \mathrm{~nm}$. Values of $E_{\text {pin }}$ and $\sigma_{\text {pin }}$ which do not improve $r_{\text {med }}$, compared to a case of no pinning potential, are left blank. (c) $2 \mathrm{D}$ representation of the pinning potential $E_{\text {pin,all }}$ for $E_{\text {pin }}=80 \mathrm{meV}$ and $\sigma_{\text {pin }}=11 \mathrm{~nm}$.

The equilibrium core position in the absence of pinning, i.e., the minimum of $E_{\text {quad }}$, is given by

$$
\left(\begin{array}{c}
X \\
Y
\end{array}\right)=c \chi\left(\begin{array}{c}
-H_{y} \\
H_{x}
\end{array}\right)=c \underbrace{\chi H_{z}}_{=: \tilde{\chi}}\left(\begin{array}{c}
-\alpha_{x} \\
\alpha_{y}
\end{array}\right) .
$$

Here we replace $\chi$ with a modified displacement susceptibility $\tilde{\chi}=\chi H_{z}$, which specifies the core movement per tilt angle. By fitting Eq. (3) to our data, we find $\tilde{\chi}$ to be 98 and $108 \mathrm{~nm} / \mathrm{deg}$ for samples $\mathbf{N} \mathbf{1}$ and $\mathbf{A}$, respectively. It is worth noting that the effects of the short-range pinning sites are negligible for the overall core displacement used in this fit. The results are in good agreement with the micromagnetic simulation, which predicted a value of $\tilde{\chi}=123 \mathrm{~nm} / \mathrm{deg}$.

A single TRaPS simulation is fed with the same sequence of tilt angles as in the experiment. At each tilt step $i$, we find the next local minimum $\left(X_{i, \text { sim }}, Y_{i, \text { sim }}\right)$ of $E_{\text {total }}=E_{\text {quad }}+$ 
$E_{\text {pin,all }}$ in the direction of the steepest descent. This is done using a numerical gradient descent minimization, where we supply the algebraic form of the gradient and the Hessian matrix of $E_{\text {total }}$ for maximum robustness. The starting point of each minimization is the simulated minimum from the previous tilt step $i-1$, just like in the experiment. The result of a TRaPS simulation is a set of simulated core positions $\left(X_{i, \mathrm{sim}}, Y_{i, \mathrm{sim}}\right)$, which we can compare to the experimental data using the median radial deviation

$$
r_{\text {med }}=\operatorname{median}_{i}\left(\| \begin{array}{c}
X_{i, \text { sim }}-X_{i, \text { expt }} \| \\
Y_{i, \text { sim }}-Y_{i, \text { expt }} \|
\end{array}\right) \text {. }
$$

Figure 5(b) shows the median radial deviation for all TRaPS simulations based on the data of sample N1. The values of $E_{\text {pin }}=80 \mathrm{meV}$ and $\sigma_{\text {pin }}=11 \mathrm{~nm}$ best reproduce the experimental data, in that they result in the smallest value of $r_{\text {med }}$. In case of sample $\mathbf{A}$, we obtain similar values of $E_{\text {pin }}=$ $90 \mathrm{meV}$ and $\sigma_{\text {pin }}=12 \mathrm{~nm}$, which results in an increase of the integrated pinning potential of about $30 \%$. The simulated core positions are in good agreement with experimental data, as we present in the Supplemental Material Fig. 9, together with images of the pinning potential [40].

A three-dimensional representation of $E_{\text {pin,all }}$ of sample N1 is given in Fig. 5(c). It has a roughness, estimated by the standard deviation of the potential, of $193 \mathrm{meV}$ and measures $-2.0 \mathrm{eV}$ at its deepest point. For the annealed sample, the respective values are $281 \mathrm{meV}$ and $-2.4 \mathrm{eV}$. This analysis demonstrates that polycrystalline samples with larger grains show an overall increase in their pinning potential, with an increased roughness and deeper minima. Furthermore, we note that the demonstrated method is capable of quantifying pinning potentials down to sub $100 \mathrm{meV}$ and spanning multiple orders of magnitude.

\section{Time-resolved trajectories}

Besides studying the static interaction of the vortex core with pinning sites, we also probed how the dynamic vortex gyration is affected by the nanocrystalline configuration. $\mathrm{We}$, therefore, perform time-resolved measurements on the nonannealed sample $\mathbf{N} \mathbf{2}$ and the annealed sample $\mathbf{A}$, where we excite the permalloy square with an alternating current forcing the vortex core on an elliptical trajectory. We recorded this motion in sample $\mathbf{N} \mathbf{2}$ for excitation frequencies from $f_{\text {ex }}=86$ to $99.5 \mathrm{MHz}$ at a current density of $j=3.6 \times 10^{10} \mathrm{~A}_{\mathrm{rms}} \mathrm{m}^{-2}$ and in case of sample $\mathbf{A}$ for frequencies from $72-84 \mathrm{MHz}$ at $j=8.2 \times 10^{10} \mathrm{~A}_{\mathrm{rms}} \mathrm{m}^{-2}$. The higher currents and lower frequencies in the case of sample $\mathbf{A}$ were only necessary after annealing, whereas before, we were able to observe core gyration at similar excitation parameters as for sample N2. To measure and ensure a constant excitation current throughout the frequency range, we monitor the sample current $I_{\mathrm{S}}$ using an oscilloscope (for details see the Supplemental Material Note $1[40])$.

At each frequency we record up to 60 micrographs and incrementally increase the phase between the RF current and the probing electron beam to cover the whole excitation period. These micrographs are combined into Supplemental Movies 4 and 5, and show the time-resolved gyration together with
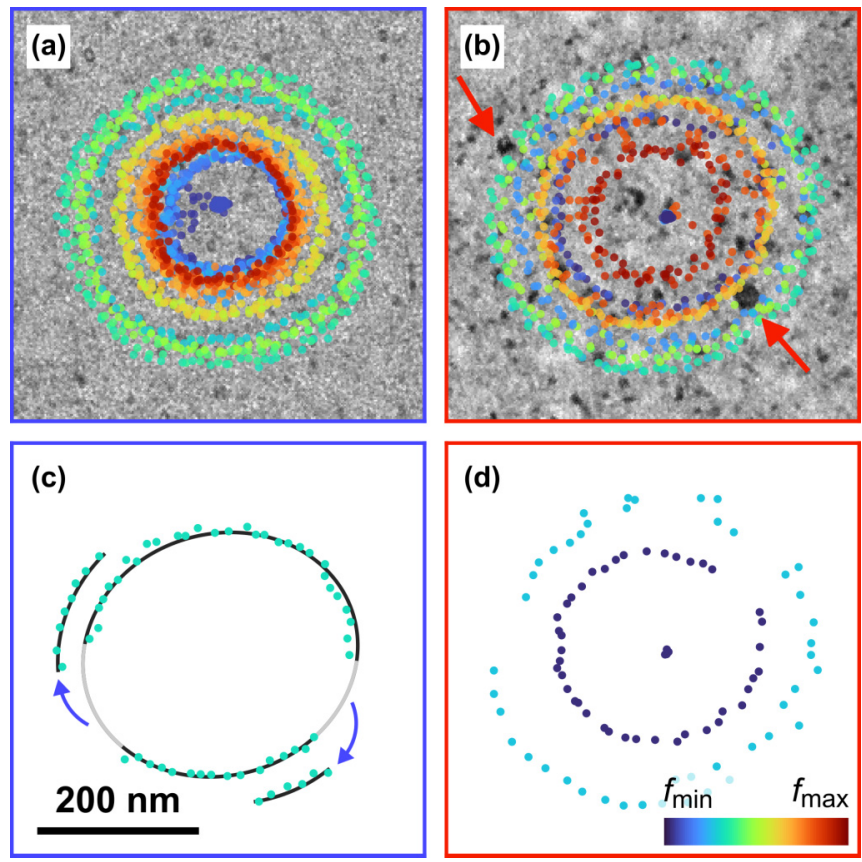

FIG. 6. (a) and (b) Trajectories (dots) of the vortex gyration in sample N2 (a) and sample A (b) measured via time-resolved Lorentz microscopy overlaid on top of bright-field images of samples. (c) Example of a segmented trajectory in (a) indicating a bistable gyration. The black line is a guide to the eye, indicating the movement of the core. The grayed-out path and the arrows indicate where the core jumped to another equally favorable orbit. (d) Two examples for jagged trajectories in (b).

the tracked core positions [40]. Figures 6(a) and 6(b) show a compilation of all trajectories overlaid on the bright-field images (individual depictions in the Supplemental Material Figs. 7 and 8 [40]).

Similar to our TRaPS measurements, we can also identify grains in the annealed sample in Fig. 6(b) that appear to be avoided by the core, however, less conclusively as in the static case. We marked two of these grains with arrows in Fig. 6(b). Furthermore, we find discontinuous jumps of the vortex position upon cycling the excitation phase, where it appears to switch between two or more equally favorable trajectories. The fact that our stroboscopic method can resolve a single vortex core before and after a jump indicates that these do not happen randomly during an image acquisition but rather in between. This suggests the phase discontinuity upon a parameter change (excitation frequency or phase) as a cause for such events. Interestingly, this behavior is encountered much more regularly for the annealed sample, both at a larger number of frequencies and during a measurement at a given frequency (see the Supplemental Material Figs. 7 and 8 [40]). This multistability can be considered the dynamic counterpart of stochastic switching between bistable static pinning sites $[24,30,58]$.

To further evaluate the trajectories, we fit them to an ellipse and determine the mean of both semiaxes $r$ [59]. Figure 7 shows the resulting radii $r$ divided by the sample current $I_{\mathrm{S}}$ and plotted against the normalized frequency $f_{\mathrm{ex}} / f_{\mathrm{r}}$. The graphs reveal a resonance frequency of the gyration of $f_{\mathrm{r}}=$ 


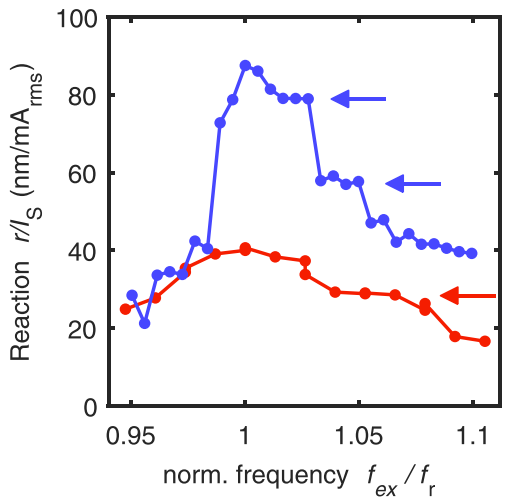

FIG. 7. Excitation-current-normalized radius $r / I_{\mathrm{S}}$ as a function of normalized frequency $f_{\mathrm{ex}} / f_{\mathrm{r}}$. For some frequency ranges the orbit radius stays constant upon changing the excitation frequency (arrows).

90.5 and $76 \mathrm{MHz}$ for samples $\mathbf{N 2}$ and $\mathbf{A}$, respectively [60]. Here two observations stand out. First, the radius of the trajectories does not change continuously in size as a function of frequency $f_{\text {ex }}$ (see also Refs. [37,43]), suggesting hysteretic behavior. Instead, we find plateaus, for which trajectories cluster at certain radii (marked with arrows in Fig. 7), clearly indicating an orbital stabilization by the pinning potential. Second, we find that the current-normalized radius $r / I_{\mathrm{S}}$ is significantly smaller and exhibits a broader resonance in the case of the annealed sample $\mathbf{A}$, which is a distinct sign of enhanced dissipation in this sample. Such enhanced dissipation can be attributed to the excitation of additional internal degrees of freedom of the vortex configuration due to the presence of defects [61]. These two observations, together with enhanced multistability in the annealed sample, demonstrate that grain sizes have an important influence on the dynamic behavior of gyrating vortices.

\section{CONCLUSION}

Nanocrystallinity and surface roughness have long been linked to the pinning of vortices in soft-magnetic films $[21,27,29]$. The direct real-space identification of grain boundaries as effective pinning sites for the core was enabled by the TRaPS method introduced in this study. The correlation of structural and magnetic imaging in electron microscopy can be further developed to trace the microscopic origins of pinning down to the atomic scale, combining high-resolution (scanning) TEM with holography $[62,63]$ or differential phase contrast $[33,64]$.

The joint high spatial and temporal resolution of our approach will be critical to explore transient pinning and local damping effects, while the quantitative TRaPS potential will serve as input for future theoretical studies on driven vortex dynamics. The global ansatz for the calculation of the trapping potential based on common properties of pinning sites may be further refined by taking into consideration characteristics of individual defects. The observation of increased roughness, deeper traps, and enhanced bistability for samples with larger grains may become relevant for device fabrication and in the tailoring of annealing processes to mitigate or selectively enhance pinning.

Finally, on the methodological side, the two orders of magnitude increase in time-averaged brightness of the photoemission source will have immediate benefits in picosecond stroboscopic imaging of ultrafast dynamics also beyond magnetism, including nanoscale structural and electronic phenomena.

\section{ACKNOWLEDGMENTS}

This work was funded by the Deutsche Forschungsgemeinschaft (DFG) in the Collaborative Research Center "Atomic Scale Control of Energy Conversion" (DFG-SFB 1073, project A05) and via resources from the Gottfried Wilhelm Leibniz-Prize. We gratefully acknowledge support by the Lower Saxony Ministry of Science and Culture and funding of the instrumentation by the DFG and VolkswagenStiftung. Furthermore, we acknowledge helpful discussions and assistance from the Göttingen UTEM Team, especially Thomas Danz, Till Domröse, Armin Feist, and Karin Ahlborn.

C.R. and M.M. conceived the project. M.M. prepared the sample. M.M. and J.H.G. conducted the experiment. M.M. evaluated the data and discussed the results with J.H.G. and C.R. M.M. and C.R. wrote the paper with inputs from J.H.G.
[1] A. Hubert and R. Schäfer, Magnetic Domains: The Analysis of Magnetic Microstructures (Springer, Berlin, 1998).

[2] T. Shinjo, T. Okuno, R. Hassdorf, K. Shigeto, and T. Ono, Magnetic vortex core observation in circular dots of permalloy, Science 289, 930 (2000).

[3] T. Eggebrecht, M. Möller, J. G. Gatzmann, N. Rubiano da Silva, A. Feist, U. Martens, H. Ulrichs, M. Münzenberg, C. Ropers, and S. Schäfer, Light-Induced Metastable Magnetic Texture Uncovered by in situ Lorentz Microscopy, Phys. Rev. Lett. 118, 097203 (2017).

[4] T. Skyrme, A unified field theory of mesons and baryons, Nucl. Phys. 31, 556 (1962).

[5] S. Mühlbauer, B. Binz, F. Jonietz, C. Pfleiderer, A. Rosch, A. Neubauer, R. Georgii, and P. Boni, Skyrmion lattice in a chiral magnet, Science 323, 915 (2009).
[6] X. Z. Yu, Y. Onose, N. Kanazawa, J. H. Park, J. H. Han, Y Matsui, N. Nagaosa, and Y. Tokura, Real-space observation of a two-dimensional skyrmion crystal, Nature (London) 465, 901 (2010).

[7] S. S. P. Parkin, M. Hayashi, and L. Thomas, Magnetic domainwall racetrack memory, Science 320, 190 (2008).

[8] N. S. Kiselev, A. N. Bogdanov, R. Schäfer, and U. K. Rößler, Chiral skyrmions in thin magnetic films: New objects for magnetic storage technologies? J. Phys. D 44, 392001 (2011).

[9] A. Fert, V. Cros, and J. Sampaio, Skyrmions on the track, Nat. Nanotechnol. 8, 152 (2013).

[10] A. Fert, N. Reyren, and V. Cros, Magnetic skyrmions: Advances in physics and potential applications, Nat. Rev. Mater. 2, 17031 (2017). 
[11] H. Jung, Y.-S. Choi, K.-S. Lee, D.-S. Han, Y.-S. Yu, M.-Y. Im, P. Fischer, and S.-K. Kim, Logic operations based on magneticvortex-state networks, ACS Nano 6, 3712 (2012).

[12] X. Zhang, M. Ezawa, and Y. Zhou, Magnetic skyrmion logic gates: Conversion, duplication and merging of skyrmions, Sci. Rep. 5, 9400 (2015).

[13] J. Torrejon, M. Riou, F. A. Araujo, S. Tsunegi, G. Khalsa, D. Querlioz, P. Bortolotti, V. Cros, K. Yakushiji, A. Fukushima, H. Kubota, S. Yuasa, M. D. Stiles, and J. Grollier, Neuromorphic computing with nanoscale spintronic oscillators, Nature (London) 547, 428 (2017).

[14] S.-B. Choe, Vortex core-driven magnetization dynamics, Science 304, 420 (2004).

[15] B. Van Waeyenberge, A. Puzic, H. Stoll, K. W. Chou, T. Tyliszczak, R. Hertel, M. Fähnle, H. Brückl, K. Rott, G. Reiss, I. Neudecker, D. Weiss, C. H. Back, and G. Schütz, Magnetic vortex core reversal by excitation with short bursts of an alternating field, Nature (London) 444, 461 (2006).

[16] K. Yamada, S. Kasai, Y. Nakatani, K. Kobayashi, H. Kohno, A. Thiaville, and T. Ono, Electrical switching of the vortex core in a magnetic disk, Nat. Mater. 6, 270 (2007).

[17] S. Woo, K. Litzius, B. Krüger, M.-Y. Im, L. Caretta, K. Richter, M. Mann, A. Krone, R. M. Reeve, M. Weigand, P. Agrawal, I. Lemesh, M.-A. Mawass, P. Fischer, M. Kläui, and G. S. D. Beach, Observation of room-temperature magnetic skyrmions and their current-driven dynamics in ultrathin metallic ferromagnets, Nat. Mater. 15, 501 (2016).

[18] K. Gerlinger, B. Pfau, F. Büttner, M. Schneider, L.-M. Kern, J. Fuchs, D. Engel, C. M. Günther, M. Huang, I. Lemesh, L. Caretta, A. Churikova, P. Hessing, C. Klose, C. Strüber, C. V. K. Schmising, S. Huang, A. Wittmann, K. Litzius, D. Metternich et al., Application concepts for ultrafast laser-induced skyrmion creation and annihilation, Appl. Phys. Lett. 118, 192403 (2021).

[19] S. G. Je, P. Vallobra, T. Srivastava, J. C. Rojas-Sánchez, T. H. Pham, M. Hehn, G. Malinowski, C. Baraduc, S. Auffret, G. Gaudin, S. Mangin, H. Béa, and O. Boulle, Creation of magnetic skyrmion bubble lattices by ultrafast laser in ultrathin films, Nano Lett. 18, 7362 (2018).

[20] M. Rahm, R. Höllinger, V. Umansky, and D. Weiss, Influence of point defects on magnetic vortex structures, J. Appl. Phys. 95, 6708 (2004).

[21] T. Uhlig, M. Rahm, C. Dietrich, R. Höllinger, M. Heumann, D. Weiss, and J. Zweck, Shifting and Pinning of a Magnetic Vortex Core in a Permalloy Dot by a Magnetic Field, Phys. Rev. Lett. 95, 237205 (2005).

[22] A. Vansteenkiste, J. De Baerdemaeker, K. W. Chou, H. Stoll, M. Curcic, T. Tyliszczak, G. Woltersdorf, C. H. Back, G. Schütz, and B. Van Waeyenberge, Influence of domain wall pinning on the dynamic behavior of magnetic vortex structures: Timeresolved scanning $\mathrm{x}$-ray transmission microscopy in $\mathrm{NiFe}$ thin film structures, Phys. Rev. B 77, 144420 (2008).

[23] C. Holl, M. Knol, M. Pratzer, J. Chico, I. L. Fernandes, S. Lounis, and M. Morgenstern, Probing the pinning strength of magnetic vortex cores with sub-nanometer resolution, Nat. Commun. 11, 2833 (2020).

[24] J. A. J. Burgess, A. E. Fraser, F. F. Sani, D. Vick, B. D. Hauer, J. P. Davis, and M. R. Freeman, Quantitative magnetomechanical detection and control of the Barkhausen effect, Science 339, 1051 (2013).
[25] R. L. Compton and P. A. Crowell, Dynamics of a Pinned Magnetic Vortex, Phys. Rev. Lett. 97, 137202 (2006).

[26] R. L. Compton, T. Y. Chen, and P. A. Crowell, Magnetic vortex dynamics in the presence of pinning, Phys. Rev. B 81, 144412 (2010).

[27] T. Y. Chen, M. J. Erickson, P. A. Crowell, and C. Leighton, Surface Roughness Dominated Pinning Mechanism of Magnetic Vortices in Soft Ferromagnetic Films, Phys. Rev. Lett. 109, 097202 (2012).

[28] T. Y. Chen, A. T. Galkiewicz, and P. A. Crowell, Phase diagram of magnetic vortex dynamics, Phys. Rev. B 85, 180406(R) (2012).

[29] R. Badea and J. Berezovsky, Mapping the Landscape of Domain-Wall Pinning in Ferromagnetic Films using Differential Magneto-Optical Microscopy, Phys. Rev. Appl. 5, 064003 (2016).

[30] R. Badea, M. S. Wolf, and J. Berezovsky, Exploiting bistable pinning of a ferromagnetic vortex for nitrogen-vacancy spin control, Appl. Phys. Lett. 109, 132403 (2016).

[31] J.-S. Kim, O. Boulle, S. Verstoep, L. Heyne, J. Rhensius, M. Kläui, L. J. Heyderman, F. Kronast, R. Mattheis, C. Ulysse, and G. Faini, Current-induced vortex dynamics and pinning potentials probed by homodyne detection, Phys. Rev. B 82, 104427 (2010).

[32] C. Hanneken, A. Kubetzka, K. von Bergmann, and R. Wiesendanger, Pinning and movement of individual nanoscale magnetic skyrmions via defects, New J. Phys. 18, 055009 (2016).

[33] S. McVitie, D. McGrouther, S. McFadzean, D. MacLaren, K. O'Shea, and M. Benitez, Aberration corrected Lorentz scanning transmission electron microscopy, Ultramicroscopy 152, 57 (2015).

[34] K. B. Schliep, P. Quarterman, J.-P. P. Wang, and D. J. Flannigan, Picosecond Fresnel transmission electron microscopy, Appl. Phys. Lett. 110, 222404 (2017).

[35] N. Rubiano da Silva, M. Möller, A. Feist, H. Ulrichs, C. Ropers, and S. Schäfer, Nanoscale Mapping of Ultrafast Magnetization Dynamics with Femtosecond Lorentz Microscopy, Phys. Rev. X 8, 031052 (2018).

[36] M. Zhang, Z.-A. Li, S. Sun, P. Xu, C. Zhu, H. Tian, Z. Li, Y. Zhang, H. Yang, and J. Li, High Spatiotemporal Resolution of Magnetic Dynamics in Mn-Ni-Ga via Four-Dimensional Lorentz Microscopy Ming, Phys. Rev. Appl. 12, 034037 (2019).

[37] M. Möller, J. H. Gaida, S. Schäfer, and C. Ropers, Few-nm tracking of current-driven magnetic vortex orbits using ultrafast Lorentz microscopy, Commun. Phys. 3, 36 (2020).

[38] X. Fu, E. Wang, Y. Zhao, A. Liu, E. Montgomery, V. J. Gokhale, J. J. Gorman, C. Jing, J. W. Lau, and Y. Zhu, Direct visualization of electromagnetic wave dynamics by laser-free ultrafast electron microscopy, Sci. Adv. 6, eabc3456 (2020).

[39] G. Cao, S. Jiang, J. Åkerman, and J. Weissenrieder, Femtosecond laser driven precessing magnetic gratings, Nanoscale 13, 3746 (2021).

[40] See Supplemental Material at http://link.aps.org/supplemental/ 10.1103/PhysRevResearch.4.013027 for (i) details on the electrical setup, (ii) details on the annealing process, (iii) correlation between bright-field image contrast and pinned vortex core positions in the TRaPS measurements, (iv) supplementary figures, and (v) supplementary movies. 
[41] S. Kasai, Y. Nakatani, K. Kobayashi, H. Kohno, and T. Ono, Current-Driven Resonant Excitation of Magnetic Vortices, Phys. Rev. Lett. 97, 107204 (2006).

[42] M. Bolte, G. Meier, B. Krüger, A. Drews, R. Eiselt, L. Bocklage, S. Bohlens, T. Tyliszczak, A. Vansteenkiste, B. Van Waeyenberge, K. W. Chou, A. Puzic, and H. Stoll, TimeResolved X-Ray Microscopy of Spin-Torque-Induced Magnetic Vortex Gyration, Phys. Rev. Lett. 100, 176601 (2008).

[43] S. Pollard, L. Huang, K. Buchanan, D. Arena, and Y. Zhu, Direct dynamic imaging of non-adiabatic spin torque effects, Nat. Commun. 3, 1028 (2012).

[44] B. Krüger, A. Drews, M. Bolte, U. Merkt, D. Pfannkuche, and G. Meier, Harmonic oscillator model for current- and field-driven magnetic vortices, Phys. Rev. B 76, 224426 (2007).

[45] M. De Graef, Experimental Methods in the Physical Sciences, Experimental Methods in the Physical Sciences, Vol. 36 (CRC, Boca Raton, FL, 2001), pp. 27-67.

[46] J. Zweck, Imaging of magnetic and electric fields by electron microscopy, J. Phys.: Condens. Matter 28, 403001 (2016).

[47] A. Feist, N. Bach, N. Rubiano da Silva, T. Danz, M. Möller, K. E. Priebe, T. Domröse, J. G. Gatzmann, S. Rost, J. Schauss, S. Strauch, R. Bormann, M. Sivis, S. Schäfer, and C. Ropers, Ultrafast transmission electron microscopy using a laser-driven field emitter: Femtosecond resolution with a high coherence electron beam, Ultramicroscopy 176, 63 (2017).

[48] A. Goshtasby, Image registration by local approximation methods, Image Vision Comput 6, 255 (1988).

[49] D.-T. Ngo and S. McVitie, Visualization of vortex core polarity in NiFe nanodots by tilted Fresnel images, Ultramicroscopy 111, 1276 (2011).

[50] M. Schneider, H. Hoffmann, and J. Zweck, Lorentz microscopy of circular ferromagnetic permalloy nanodisks, Appl. Phys. Lett. 77, 2909 (2000).

[51] R. P. Heilbronner, The autocorrelation function: An image processing tool for fabric analysis, Tectonophysics 212, 351 (1992).

[52] B. Zang, K. Suzuki, and A. C. Liu, Estimation of volumeweighted average grain size in Fe-based nanocrystalline soft magnetic materials by autocorrelation function, Mater. Charact. 142, 577 (2018).

[53] It is worth noting that dark-field images were used for this assessment in the original publications. However, we believe that for a rough estimate, we are interested here, bright-field images are equally suitable.
[54] K. Y. Guslienko, V. Novosad, Y. Otani, H. Shima, and K. Fukamichi, Field evolution of magnetic vortex state in ferromagnetic disks, Appl. Phys. Lett. 78, 3848 (2001).

[55] A. Vansteenkiste, J. Leliaert, M. Dvornik, M. Helsen, F. GarciaSanchez, and B. Van Waeyenberge, The design and verification of MuMax3, AIP Adv. 4, 137133 (2014).

[56] Y. Yin, F. Pan, M. Ahlberg, M. Ranjbar, P. Dürrenfeld, A. Houshang, M. Haidar, L. Bergqvist, Y. Zhai, R. K. Dumas, A. Delin, and J. Åkerman, Tunable permalloy-based films for magnonic devices, Phys. Rev. B 92, 024427 (2015).

[57] The value of $M_{\mathrm{S}}$ was obtained from a comparison of experimental and simulated Lorentz images, using the nominal film thickness of $30 \mathrm{~nm}$. It is somewhat lower than expected from bulk values, which might be caused by uncertainty in the determination of the film thickness. Furthermore, it is worth noting that we found $k$ to be virtually unchanged under an applied field $H_{z}$ unlike the free gyration frequency, which is a function of the field [65].

[58] R. Badea, E. Haber, and J. Berezovsky, Stochastic Dynamics of a Ferromagnetic Vortex Revealed by Single-Spin Magnetometry, Phys. Rev. Appl. 10, 064031 (2018).

[59] O. Gal, fit_ellipse - File Exchange - MATLAB Central (2003).

[60] Note that the lack of a significant ellipticity in the studied frequency range agrees with expectations based on Ref. [66].

[61] H. Min, R. D. McMichael, J. Miltat, and M. D. Stiles, Effects of disorder on magnetic vortex gyration, Phys. Rev. B 83, 064411 (2011).

[62] H. Lichte and M. Lehmann, Electron holography basics and applications, Rep. Prog. Phys. 71, 016102 (2008).

[63] P. A. Midgley and R. E. Dunin-Borkowski, Electron tomography and holography in materials science, Nat. Mater. 8, 271 (2009).

[64] J. N. Chapman, P. E. Batson, E. M. Waddell, and R. P. Ferrier, The direct determination of magnetic domain wall profiles by differential phase contrast electron microscopy, Ultramicroscopy 3, 203 (1978).

[65] G. de Loubens, A. Riegler, B. Pigeau, F. Lochner, F. Boust, K. Y. Guslienko, H. Hurdequint, L. W. Molenkamp, G. Schmidt, A. N. Slavin, V. S. Tiberkevich, N. Vukadinovic, and O. Klein, Bistability of Vortex Core Dynamics in a Single Perpendicularly Magnetized Nanodisk, Phys. Rev. Lett. 102, 177602 (2009).

[66] B. Krüger, M. Najafi, S. Bohlens, R. Frömter, D. P. F. Möller, and D. Pfannkuche, Proposal of a Robust Measurement Scheme for the Nonadiabatic Spin Torque Using the Displacement of Magnetic Vortices, Phys. Rev. Lett. 104, 077201 (2010). 